\title{
FILOSOFÍA DE LA PREHISTORIA DE MÉXICO
}

\section{La cultura paleolítica}

De acuerdo con los hallazgos arqueológicos que se han hecho hasta ahora, entre los cuales no figuran restos de primates superiores o de homínidos antecesores del Homo sapiens, resulta imposible sostener científicamente un origen autóctono para el hombre americano. Por lo tanto, mientras no se hagan nuevos descubrimientos que permitan concluir otra cosa, lo más probable es considerar que los primeros humanos que poblaron América fueron grupos de inmigrantes mongoloides provenientes de Asia, que penetraron por el Estrecho de Bering aproximadamente 25000 años a.n.e., o sea, durante el periodo geológico del pleistoceno superior. Después estos grupos se fueron adentrando en el Continente americano, avanzando con lentitud y en oleadas sucesivas que duraron varios milenios, hasta llegar a poblarlo por entero. En épocas posteriores posiblemente se vinieron a sumar otros grupos - que llegaron por ese mismo camino o cruzando en balsas el Océano Pacífico - constituidos por australoides, negroides, mongoloides y polinésicos. En todo caso, los testimonios más antiguos de poblamiento humano son los restos encontrados en Tule Springs, cerca de Las Vegas, en el Estado de Nevada, para los cuales se ha determinado la fecha de 22000 años a.n.e. empleando el carbono 14. Dichos restos consisten en ruinas de hogares, huesos de camello, caballo, bisonte y mamut, una lasca de obsidiana encajada entre huesos y algunos instrumentos burdos de hueso.

En lo que respecta al México Antiguo, los primeros testimonios de la presencia del hombre se remontan a poco antes del año 9000 a.n.e. En Baja California, cerca del arroyo Comondú, se hallaron restos fósiles de bisonte, camello y caballo, con las extremidades calcinadas por agentes humanos. En Chihuahua, en la cuenca del Tildio, se encontraron instrumentos paleolíticos. En Tamaulipas, cerca del arroyo de Salinillas, se descubrieron restos de mamut asociados a una lasca de obsidiana de fabricación humana; en el arroyo de Chorreras, huesos de mamut asociados a un hogar con cenizas; 'y en las cuevas del Cañón del Diablo, un complejo que comprende varios niveles culturales, del cual hablaremos después en relación con el desarrollo evolutivo que pone de manifiesto. En Durango, algunos instrumentos encontrados al oeste del Lago de Santiaguillo y cerca de El Salto. En Guanajuato, en la Cañada del Marfil, se conoce un bisonte asociado a puntas de piedra. En Jalisco, se descubrieron artefactos líticos en las cercanias del Lago de Chapala y en el Río Juchipila. En Oaxaca se desenterraron algunos objetos paleolíticos en Mitla. En Campeche, tenemos la industria lítica de La Con- 
cepción. Y en la Cuenca de México, los restos del hombre de Tepexpan; varios artefactos de sílice y obsidiana -incluyendo una punta de proyectilencontrados en asociación indudable con un esqueleto completo de mamut y otro cráneo más, también en Tepexpan; las dos osamentas de mamut encontradas en Santa Isabel Ixtapan, en estrecha asociación con algunas puntas de proyectil; los artefactos de San Juan; y el hombre del Peñón de los Baños.

Además de los hallazgos que acabamos de mencionar, se han descubierto otros muchos correspondientes a épocas posteriores; y todos ellos muestran acusadamente la existencia de un desarrollo general común de los habitantes, en lo que respecta a sus aspectos económico, social, cultural y científico. El territorio en que se produjo la evolución de nuestra cultura indígena es conocido con el nombre de México Antiguo, y ocupó una vasta región de América del Norte y del Centro. En el norte, sus límites parten de la costa del Océano Pacífico, en el actual Estado de Sinaloa, formando una gran curva depresiva hacia el centro, que luego asciende de nuevo para abarcar la región de la Huasteca y terminar en el actual Estado de Tamaulipas, en la costa del Golfo de México. Por el sur se extiende hasta Nicaragua, aunque sus límites son menos precisos. Por lo tanto, el México Antiguo comprendía todo el territorio mexicano situado al sur de la línea apuntada, la República de Guatemala y Belice en toda su extensión, la mitad de la República de Honduras y parte de las Repúblicas de El Salvador y de Nicaragua. Recientemente, los arqueólogos y prehistoriadores le han dado a este territorio el nombre de "Mesoamérica", el cual tiene el grave inconveniente de emplear una designación aparentemente geográfica que carece de significado en geografía. Además, lejos de representar alguna ventaja connotativa o denotativa, dicho término simplemente parece representar un intento - consciente o inconsciente- de subestimación para nuestro país.

Entre los rasgos comunes que presentan las distintas variantes peculiares que tuvo el desarrollo cultural del México Antiguo, podemos citar: el calendario ceremonial de doscientos sesenta días, denominado tonalpohualli por los nahoas y tzolkin por los mayas; el calendario solar de trescientos sesenta y cinco días, cuya precisión se conseguía con correcciones semejantes a las actuales; los conocimientos astronómicos y sus interpretaciones astrológicas; la escritura jeroglífica empleada para registrar los acontecimientos y transmitir el saber; la numeración vigesimal utilizada en la astronomía y en el comercio; la similitud de su politeismo basado en el culto a la naturaleza; la estructura de su organización social y de su desenvolvimiento económico; la edificación de templos majestuosos; el dibujo, la pintura y la escultura; el uso del estuco en la arquitectura; los rasgos principales de sus concepciones estéticas; un conocimiento penetrante de los vegetales; y una medicina notablemente desarrollada. Es claro que las características acabadas de mencionar no se muestran con pleno vigor hasta la época de la cultura 
urbana o civilización y que, por otra parte, se destacaron definidamente varias culturas específicas. Pero no cabe duda de que en esos rasgos característicos en que coincidieron se advierte decididamente una relación estrecha y un paralelismo en su desarrollo, cuya formación se inició desde la época en que se produjo la revolución neolítica. Más aún, entre los pobladores del México Antiguo existe una continuidad étnica bastante acusada -aunque presente, como es natural, algunas variantes típicas - que abarca desde el hombre de Tepexpan hasta los actuales mexicanos, guatemaltecos, beliceños, hondureños, salvadoreños y nicaragüenses. $\mathrm{Y}$, por otro lado, todas las lenguas habladas en el México Antiguo tienen un origen común y una multitud de influencias recíprocas en su desenvolvimiento.

En la época paleolítica los hombres vivieron en grupos reducidos que se sustentaban mediante la caza, la pesca y la recolección. Sus utensilios fueron ramas y trozos de madera, hueso o piedra, afilados toscamente 0 adaptados con rudeza para que se acomodaran a la mano, por el procedimiento primitivo de partirlos o astillarlos. Empleando estos utensilios, los hombres paleolíticos tendían trampas y cazaban mamíferos y aves, atrapaban insectos, peces, reptiles y batracios, recolectaban granos, frutos, moluscos y huevos, extraían raices y larvas, destrozaban árboles, partían piedras y destazaban los animales cobrados. Así se hacían de carne, grasas, semillas y otros alimentos vegetales y animales, lo mismo que de pieles, astas, huesos, madera, piedras y ligamentos para sus artefactos. Sabemos que conocían el uso del fuego, se cubrian el cuerpo para aliviar los rigores del clima y construían abrigos con piedras y ramas, cuando no encontraban cuevas convenientes para su habitación. En los hallazgos correspondientes al lapso comprendido entre 20000 y 12000 años a.n.e., figuran algunos instrumentos como grabadores, raspadores y otros utensilios de dudosa diferenciación - que deben de haber tenido muchos empleos-, hechos principalmente de obsidiana y calcedonia, mediante burdas técnicas de lasqueado y astillado. En el periodo posterior, entre 12000 y 8000 años a.n.e., ya elaboraban raspadores, puntas de proyectil, lascas, núcleos, navajas, perforadores, grabadores y martillos, hechos de calcedonia, cuarzo y pedernal. Todos estos utensilios eran productos del trabajo doméstico y podían ser elaborados prácticamente por cualquiera de. los miembros del grupo, sin implicar propiamente especialización en el trabajo, ni tampoco el intercambio de unos grupos con otros; además, cada individuo podia hacer y desechar diariamente varios de estos utensilios.

En el curso de la producción de sus instrumentos, las comunidades paleolíticas empezaron a edificar una tradición científica, registrando y transmitiendo cuáles piedras eran mejores para los diversos usos, lo mismo que en dónde se encontraban y cómo se empleaban. Sólo después de dominar la técnica de su fabricación fue que el hombre pudo elaborar con éxito utensilios específicos para cada operación particular. Por otra parte, con el uso 
del fuego, el hombre consiguió el dominio de una energía física poderosa y un agente químico sumamente activo, dando el primer gran paso en la emancipación de su servidumbre del medio ambiente. Encendiendo y alimentando el fuego, transportándolo y utilizándolo, el hombre se desvió revolucionariamente del comportamiento de los otros animales, afirmando su humanidad y comenzando su evolución social. El mantenimiento del fuego sagrado y las ceremonias impresionantes que se celebraban cada cincuenta,y dos años en el México Antiguo, para hacerlo surgir nuevamente, son reminiscencias de la época en que el hombre todavía no aprendia a producir fuego a voluntad. Con el dominio del fuego, el hombre se convirtió conscientemente en un creador. Ahora bien, para tener éxito en sus actividades, el hombre tuvo que adquirir por experiencia un conjunto considerable de conocimientos astronómicos, geológicos, botánicos y zoológicos; y en la adquisición y la comunicación de estos conocimientos se fueron estableciendo las bases de la ciencia. Igualmente, los hombres aprendieron a actuar en compañía y cooperando unos con otros para conseguir la realización de sus propósitos. Particularmente en la caza del mamut se puede advertir claramente cómo ésta se lograba únicamente mediante la cooperación de un grupo numeroso de hombres, que planeaban su acción con base en el conocimiento detallado de los hábitos de las manadas.

La organización social del hombre paleolítico debe de haber sido la comunidad igualitaria, formando pequeños grupos cuyo crecimiento estaba limitado inexorablemente por el abastecimiento alimenticio disponible, lo mismo que por la forma aleatoria de procurarse la subsistencia; además, sus campamentos tenían que cambiar con alguna frecuencia, para seguir los desplazamientos de las manadas. Su tradición tecnológica la importaro de Asia y es muy probable que también hayan traído ya domesticado al perro, dada la gran dispersión de este animal en América y su gran número de variedades. Su régimen económico fue sumamente conservador y de prolongada duración. Desde luego, las comunidades paleolíticas fueron autosuficientes, pero no estuvieron completamente aisladas, sino que practicaron el comercio en forma rudimentaria y ocasional. Con todo, la recolección de alimentos ofreció muchas más posibilidades de las que generalmente se piensa. Aunque no se introdujo ningún cambio fundamental en la técnica, ni menos en la economía, sin embargo se mejoraron mucho los procedimientos de recolección y los hombres paleolíticos aprendieron a discriminar mucho mejor lo que podían recoger o extraer. A la vez, consiguieron fabricar muchos artefactos distintos adaptados a usos particulares, elaboraron incluso instrumentos para hacer instrumentos, trabajaron el hueso con la misma habilidad que el pedernal, e inventaron un artefacto mecánico simple, el átlatl o lanzadera, con el cual multiplicaron ingeniosamente la energía muscular del hombre aprovechando la ley de la palanca. La fabricación de estos nuevos 
instrumentos no sólo indica un incremento en la destreza técnica, sino una acumulación mayor de conocimientos y una aplicación más amplia de la ciencia.

\section{La revolución neolitica}

La revolución neolítica se caracterizó por la iniciación de la agricultura. El hombre fue acumulando pacientemente sus observaciones acerca del desarrollo de las plantas y advirtió también su crecimiento cuando los granos quedaban abandonados en las cercanías de sus albergues. Finalmente se decidió a intervenir en el proceso y comenzó a sembrar, cultivar y mejorar por selección algunas yerbas, raices y arbustos comestibles. Desde luego, todas las plantas cultivadas son formas domesticadas de especies silvestres y, por lo tanto, representan propiamente una creación humana. Con la agricultura se produjo una transformación radical en la economía, ya que permitió al hombre el dominio sobre su abastecimiento alimenticio. El hombre se convirtió así en productor y se aseguró la satisfacción de sus necesidades primordiales. La economía productora de alimentos afectó profundamente la existencia humana. Entonces comenzó la vida sedentaria, estableciéndose pequeños poblados rurales permanentes. Además, al romper las limitaciones de la economía recolectora, la agricultura propició el crecimiento de la población humana en una forma muy considerable. Sin embargo, la agricultura no desalojó por entero a la recolección, ni menos lo hizo bruscamente, sino que la complementó durante mucho tiempo. En el México Antiguo todavía en 1521 la montería, la volatería y la recolección de frutos, raíces, insectos y moluscos seguían siendo actividades indispensables para la alimentación. En realidad, sólo muy lentamente la agricultura llegó a conquistar una posisión independiente, que hasta mucho más tarde se convirtió en predominante. Por otro lado, poco a poco se fueron incorporando a las tareas agrícolas más y más mujeres, luego participaron también los niños - quienes se hicieron así económicamente útiles, por primera vez-y, por último, tomaron parte los varones; hasta que, después de un lapso bastante prolongado, la agricultura acabó por ser la actividad económica preponderante. $\mathrm{Y}$, como es sabido, este predominio de la población rural ha perdurado en las sociedades humanas hasta el siglo XIx, en los paises más desarrollados industrialmente, y hasta nuestros dias en el resto del mundo.

Con la agricultura se produjo un aumento en la productividad del trabajo humano, permitiendo que por primera vez hubiera un excedente entre lo producido y lo consumido por los productores. A la vez, la producción de alimentos, aun en su forma más simple, impuso la necesidad de construir recintos para el almacenamiento de las cosechas; porque no se consumía todo desde luego, sino que se requería conservar y escatimar los granos para que 
durasen hasta la siguiente cosecha y, por otra parte, era preciso apartar la semilla para la siguiente siembra. Esto hizo que se desarrollara la previsión y la administración de los abastecimientos. Por otro lado, el almacenamiento constituyó una base para el comercio rudimentario. Desde el punto de vista tecnológico, la revolución neolítica se puso de manifiesto con los instrumentos de piedra pulimentada, que aumentaron enormemente la eficacia de la actividad práctica del hombre y ensancharon sus posibilidades. A más de esto, se produjo un perfeccionamiento importante en el arte de cocinar. De esta manera se fue imponiendo la vida sedentaria, aunque tal cosa no fue necesariamente contemporánea de la nueva economía. En realidad, algunas tribus cazadoras y pescadoras llegaron a hacerse sedentarias; mientras que hubo tribus agricultoras que mantuvieron el nomadismo -y de ello tenemos muchos ejemplos en el México Antiguo-, cuando los procedimientos primitivos de cultivo conducían al agotamiento del suelo. El desenvolvimiento de la agricultura trajo consigo el establecimiento de comunidades cada vez más numerosas y económicamente autosuficientes, pero en un nivel superior al de las paleolíticas; puesto que cada comunidad producía y recogía sus alimentos, tenía a su disposición en la vecindad inmediata las materias primas requeridas para la satisfacción de todas sus necesidades, y sus miembros fabricaban los utensilios, instrumentos y armas que empleaban. Pero esta autosuficiencia no representó necesariamente un aislamiento. En realidad, durante la época neolítica el México Antiguo era -al igual de lo que ocurrió en otras regiones del mundo- una cadena continua de comunidades que se encontraban en contacto recurrente, aunque éste no fuera muy frecuente ni se hiciera de manera regular. En este sentido, lo que se destaca en la arqueología son algunas fases transitorias - cuyos vestigios se han conservado por diversas circunstancias favorables_ dentro de lo que fue un proceso continuo con un desenvolvimiento evolutivo. Por esto se ha hecho la distinción de una asombrosa variedad de culturas neolíticas, cuyas diferencias se explican por la autosuficiencia de las comunidades, la relativa independencia de su desarrollo y, sobre todo, por la carencia de datos acerca de sus interrelaciones.

La revolución neolítica está representada específicamente en el México Antiguo por los llamados complejos de Chalco y de Chupicuaro, cuya duración se calcula entre los años 6000 y 3000 a.n.e. En la Cuenca de México la caza mayor empezó a escasear, hasta que desapareció por completo. Se considera que a partir del año 4000 a.n.e., las condiciones climáticas de dicha Cuenca se han mantenido aproximadamente iguales a las que ahora existen. Entre los objetos hallados en esos sitios se tienen raspadores ovoides y discoides, martillos de mano y metates rudimentarios, hechos de andesita, basalto y obsidiana, lo mismo que instrumentos cortantes y punzantes, y figuras de animales trabajadas en hueso. Los mejores testimonios conocidos 
del desarrollo tecnológico se encuentran en unas cuevas de la Sierra Madre Oriental, en el Estado de Tamaulipas, según ya lo habíamos mencionado. Las culturas denominadas "Diablo" y "Lerma" corresponden al predominio de la caza. La cultura "Nogales", representada por utensilios de piedra -incluyendo morteros y molinos de mano- indica un cambio a formas de vida semisedentarias, basadas en la recolección y la caza. La cultura de "La Perra" corresponde a una economía agrícola primitiva que empieza a abrirse paso entre la recolección todavía predominante y la caza. De acuerdo con los restos hallados en los depósitos, la importancia relativa de los alimentos era la siguiente: $10 \%$ de caza mayor, $86 \%$ de plantas silvestres e insectos, y $4 \%$ de calabaza y maíz cultivados. Entre los utensilios hay molinos de mano, cestas, esteras y redes, pero no existe aún cerámica. En otra excavación posterior se han encontrado niveles culturales semejantes hasta la cultura de "La Perra", con una dieta formada principalmente por plantas silvestres; luego aparece un tipo primitivo, en un periodo todavía anterior a la cerámica; y, finalmente, en el nivel inmediato se encuentra ya la alfarería, junto con el maíz híbrido, restos de tejidos de algodón y algunas figurillas de cerámica.

\section{La cultura agricola}

La agricultura en el México Antiguo se basó en el sistema de reproducción de las semillas; a diferencia del sistema de reproducción vegetativa utilizado en la región incaica, que es más amplio y variado, y en donde se llegó a superar la simple técnica de la explotación del suelo, reconstruyendo su fertilidad mediante el uso del guano. En todo caso, el cultivo requirió la observación cuidadosa de las estaciones, con la consiguiente división más precisa del tiempo y la determinación del año. Las faenas agrícolas son fundamentalmente de temporada y su éxito depende mucho de la oportunidad con que se ejecutan. En las regiones tropicales, los cambios en el curso del sol no son muy notables para indicar las estaciones y, por ello, los habitantes del México Antiguo recurrieron al movimiento de las estrellas - siempre visibles en nuestros cielos despejados_- para determinar el año solar y dividirlo convenientemente. Con la observación precisa de que ciertas estrellas ocupan una posición destacada en la época de la siembra, y otras lo hacen cuando se avecinan las lluvias, surgió la astronomía; y, al mismo tiempo, se inició también la astrología, por la explicable confusión entre la conexión temporal y el enlace causal entre los fenómenos celestes y los terrestres. Por otra parte, la tejeduría requirió del conocimiento de materiales especiales como el algodón y la práctica de su cultivo específico, además de la invención de algunos instrumentos complejos, como el telar y el torno de hilar. Y tanto la artesanía textil como los otros oficios neolíticos se apoyaron en 
un conjunto de conocimientos científicos prácticos, que se ampliaban constantemente. Las inferencias correctas extraídas de la experiencia se encontraban mezcladas con un buen número de hechizos y ritos; y este conjunto de reglas prácticas y mágicas formaba la tradición del oficio, que se transmitía de padres a hijos y de generación en generación, por medio del ejemplo y del precepto.

En lo que se refiere a la domesticación de animales, es bien sabido que en Asia, Europa y África se inició inmediatamente después de la agricultura y se desarrolló en forma casi paralela a ella. En cambio, en el México Antiguo los animales domesticados fueron unos cuantos: el guajolote, el perro -incluyendo una variedad comestible_, el pato, la paloma, la codorniz, la abeja y, probablemente, el ganso. Esto se debió al hecho de que en la época en que se efectuó la revolución neolítica ya habían desaparecido las grandes especies herbívoras que fueron susceptibles de dòmesticación en otros Continentes. Tal vez esta carencia de grandes animales domesticados ha sido una de las causas que han provocado confusión entre algunos arqueólogos, prehistoriadores y protohistoriadores, quienes, desconcertados por la inexistencia de la ganadería, dudan incluso de que se haya producido la revolución neolítica. Ahora bien, en realidad, los habitantes del México Antiguo supieron encontrar en el mundo vegetal prácticamente todo lo que hubieran necesitado de los animales, adquiriendo consecuentemente los amplios conocimientos botánicos de los cuales se maravillaron los europeos, y aplicándolos con bastante acierto. Por otra parte, es pertinente recordar que las especies animales domesticadas en el Viejo Mundo no fueron muy variadas; reduciéndose esencialmente al ganado vacuno, caprino, ovino y porcino, a los cuales se agregaron solamente después, como especies importantes, la gallina y el ganado caballar. Más aún, en el centro de Europa y en el occidente de China, en donde ha prevalecido tradicionalmente la conjugación de la agricultura y la ganadería, los arqueólogos han encontrado que sus habitantes neolíticos contaban con muy pocos animales, cuando efectivamente tenían algunos; por lo cual debemos inferir que vivían fundamentalmente de los productos agrícolas, complementados todavía con la caza.

El almacenamiento de los cereales y la preparación de alimentos requirió la fabricación de vasijas que pudieran contener líquidos calientes. Así surgió la alfarería, que es una característica universal de las comunidades neolíticas. Esta nueva industria tuvo gran importancia para el desarrollo del pensamiento humano y para el comienzo de la ciencia. La elaboración de objetos de arcilla cocida se basa en la utilización consciente de una transformación química relativamente compleja. El proceso consiste fundamentalmente en expulsar, por medio del calor, el "agua de constitución" combinada químicamente con el silicato de aluminio hidratado, que es la arcilla de los alfareros. La arcilla húmeda es completamente plástica, pero al calentarla hasta una temperatura de unos $600^{\circ}$, se consigue expulsar el agua de constitución y la arcilla pierde 
definitivamente su plasticidad, conservando entonces su forma rígidamente ya sea que se encuentre húmeda o seca, e incluso puede ser sometida nuevamente al calor sin que se afecte. Aprovechando estas propiedades se pueden modelar objetos de cualquier forma deseada que, después de cocidos, sólo se destruyen al romperse deliberadamente o por accidente. La alfarería estimuló en el pensamiento humano la consideración de que el hombre es creador, puesto que puede producir formas de una masa informe; aunque, en la práctica, dicha libertad se encuentra condicionada por el hecho de que la imaginación no puede trabajar partiendo estrictamente de la nada, sino que tiene que crear siempre con base en algo conocido. Entre los objetos de arcilla cocida que no eran propiamente utensilios, hay una notable profusión de pequeñas figurillas femeninas con los rasgos sexuales muy acusados, que representaban a las "diosas de la fecundidad", las cuales se labraban antes trabajosamente en piedra. Y entre las invenciones necesarias para la tejeduría, es importante el torno de hilar - que todavía se emplea en algunas comunidades indigenas de México-, en el cual se usan pequeños discos de arcilla cocida que sirven como volantes en miniatura, para mantener el movimiento de rotación que va enrollando el hilo.

Los oficios neolíticos siguieron siendo domésticos, sin que hubiera propiamente una especialización, sino simplemente una división del trabajo por sexos y edades. Sin embargo, las tradiciones de los oficios no eran individuales, sino colectivas; ya que la economía neolítica en su conjunto no podia existir sin el esfuerzo cooperativo. Estas condiciones implicaron una cierta organización social, para controlar y coordinar las actividades de la comunidad. Las nuevas fuerzas dominadas por el hombre - como resultado de la revolución neolítica y de los conocimientos obtenidos y aplicados en el ejercicio de los nuevos oficios- deben de haber afectado notablemente la organización y el pensamiento humanos, haciendo que se modificaran sus instituciones y se transformaran sus ideas mágicas y religiosas. En todo caso, la vida siguió siendo muy precaria para los pequeños grupos de campesinos autosuficientes, ya que bastaba una sequía, una granizada de consideración o una plaga, para que se perdieran las cosechas y se produjera el hambre, dado que las reservas almacenadas no eran muy grandes y, lo que es más, porque una misma catástrofe podía destruir todos sus cultivos. El hombre dependía directamente de la lluvia, el sol, la tempestad, el huracán y las heladas; por lo cual consideró necesario halagar, propiciar o ahuyentar las fuerzas que las producen. $\mathrm{Y}$ así se desarrollaron vigorosamente las magias, los mitos y las ceremonias rituales.

La época neolítica se caracterizó tecnológicamente por el desenvolvimiento y la generalización de los instrumentos de piedra pulimentada; por la evolución de la agricultura, hasta quedar convertida en la actividad predominante; por el surgimiento y el desarrollo de la alfarería; por la iniciación de los teji- 
dos de algodón; por un considerable incremento de la población, que se agrupó en comunidades rurales; y, posiblemente, por el comienzo de la horticultura. No obstante, como ya lo dijimos, la recolección de yerbas, frutos, raices e insectos siguió siendo una fuente importante de abastecimiento alimenticio. La organización social fue tal vez el clan matrilineal, aunque no es posible asegurar que todas las comunidades tuvieron esa organización. La población de algunos sitios conocidos - como El Arbolillo I, Tlatilco Inferior y Zacatenco Inferior - era de unos 200 habitantes por comunidad. Por lo demás, no existió propiamente una "cultura neolítica", sino una multitud de aplicaciones concretas diferentes de unas cuantas técnicas y nociones generales. Posiblemente la carencia de ideologías rígidas y de instituciones sociales profundamente arraigadas permitió el progreso rápido de las poblaciones rurales; ya que, como se sabe, las instituciones firmemente establecidas y las supersticiones mantenidas con pasión, son notablemente hostiles a la transformación de la sociedad y a los avances científicos que la hacen necesaria. Debido a este rápido progreso, la época neolítica propiamente dicha tuvo una duración relativamente corta, y muy pronto surgieron los primeros elementos de la revolución urbana. En el México Antiguo, la época neolitica puede situarse entre los años 3000 y 900 a.n.e. aproximadamente, con un gran número de variantes en los diversos sitios. De hecho, se trata de una de las épocas de las cuales se tienen menos datos arqueológicos. En ella quedan comprendidos el llamado Periodo Premaya I, el Arcaico o Primitivo de otras regiones, y la mayor parte de la llamada Etapa Protoagrícola. Y en el seno de estas comunidades tan poco conocidas por nosotros, pero que tuvieron bastante homogeneidad, fue en donde surgieron los factores de su transformación en poblaciones civilizadas.

\section{La revolución urbana}

La época de la revolución urbana en el México Antiguo abarca los periodos llamados Arcaico y Formativo, las culturas Preclásicas, los periodos Premaya II y III, y la última parte de la Etapa Protoagrícola junto con la primera parte de la Etapa de las Civilizaciones. Cronológicamente la podemos fijar entre los años 900 a.n.e. y 400 de nuestra era; aunque siempre tomando en cuenta que las fechas se refieren a las regiones más adelantadas, y sin olvidar que en otras partes siguen subsistiendo durante mucho tiempo las comunidades neolíticas. Al principio de esta época se puede advertir claramente la coexistencia de ambas culturas en forma bastante bien definida. Por una parte encontramos las pequeñas comunidades rurales que basan su economía en la agricultura, con poblados pequeños y diseminados, y cuyos habitantes seguían elaborando utensilios de obsidiana, hueso y piedra volcánica, y haciendo piezas de alfarería doméstica y figurillas femeninas de arcilla. Esta 
cultura neolítica se mantuvo con mayor persistencia en los valles y las tierras altas templadas. Por otro lado, empezaron a surgir indicios de la transformación de esas comunidades igualitarias de agricultores, debido al perfeccionamiento de las técnicas, la agricultura intensiva, el desarrollo de nuevos instrumentos tecnológicos, la división del trabajo, la acumulación de riquezas debido al aumento de la productividad y la formación de una clase dirigente que se apropiaba de dichas riquezas. Estos elementos se desarrollaron primero en las regiones tropicales y semitropicales de ambos litorales; y, por lo tanto, también fue en dichas regiones donde se realizó en primer lugar la revolución urbana, en la modalidad conocida con el nombre de cultura olmeca. Y de allí fue de donde se propagó a las tierras altas, cuando se consiguió adaptar el maíz para su cultivo en los Altos de Guatemala, la Meseta de Chiapas y las Cuenca de México.

Los principales testimonios arqueológicos correspondientes a la época de la revolución urbana en el México Antiguo son los que indicamos a continuación. En la Cuenca de México: E1 Arbolillo II, Tlatilco Superior, Zacatenco Medio y Superior, Copilco, Atoto, Coatepec, Xaloztoc, Lomas de Becerra, Naucalpan, Azcapotzalco, Tetelpan, Ticomán, Cuicuilco, Cerro del Tepalcate, Teotihuacán I, Tlapacoya, Ecatepec, Contreras, Cerro de la Estrella, Tepetlaoztoc, Chimalhuacán, Papalotla y San Sebastián. En el Estado de Morelos: Gualupita I y II, Chalcatzingo, Atlihuayán y Tlaltizapán. En el Estado de Oaxaca: Monte Albán I y II, Valle de Oaxaca, Monte Negro y la región mixteca. En el Estado de Veracruz: Tres Zapotes Inferior y Superior, El Trapiche, y la región del Pánuco. En el Estado de Michoacán: El Opeño y Chupicuaro. En el Estado de Tabasco: La Venta. En el Estado de Tamaulipas: Pavón I y II. En el Estado de México: el Valle de Toluca. En el Estado de Guerrero: la región costera. En el Estado de Puebla: el Valle de Puebla. En los estados de Sinaloa, Nayarit, Jalisco y Colima: los sitios correspondientes a las llamadas culturas de occidente. En la República de Guatemala: las fases Mamon y Chikanel de Uaxactún, y los periodos Majadas, Arévalo y Providencia-Sacatepequez de Kaminaljuyú. Y en la República de Honduras: el sitio conocido con el nombre de Playa de los Muertos.

Durante la época de la revolución urbana en el México Antiguo se em. plearon materiales como la piedra volcánica, la obsidiana, el pedernal, el cuarzo, la jadeíta, la serpentina, el jade, la arcilla, el caolín, las astas y huesos de venado, las conchas, maderas de diversas clases y fibras vegetales. En cada región, algunos de estos materiales eran nativos, pero otros tenían que obtenerse de distintas partes y, por lo tanto, implicaron el establecimiento de un intercambio comercial a base de trueque. Lo que resulta particularmente característico es el trabajo de las piedras duras, la elaboración de una cerámica de tipo ritual y funerario —además de la doméstica- y la introducción de las vasijas de caolin, el asa de estribo y la pintura estucada. 
Con los materiales mencionados los indígenas elaboraron entonces mazas, bolas, puntas de armas arrojadizas, bastones para sembrar, azadas, punzones, leznas, agujas, taladros, cinceles, hachas, cuchillos, navajas, raederas, buriles, raspadores, pulidores, molcajetes y sus correspondientes manos, metates y sus metlapil, piedras-yunques, cestas, redes, lazos, dardos, átlatl, arcos, hondas, telas de algodón y yuca, grandes vasijas de arcilla cocida para almacenar agua y alimentos, vasijas menores para cocinar, vasos, incensarios, copas, sahumerios, vasijas ornamentadas, figurillas rituales, platos, botellones, jarros y cucharas. Las habitaciones eran construidas con adobes, troncos, cañas, tule, ramas y paja, de tal manera que no han quedado muchos vestigios de ellas; pero las ringleras de piedras, los restos de pavimentos y otras ruinas que se han conservado indican que las habitaciones eran de forma rectangular, con troncos hincados en el suelo, muros de varas entretejidas con tules y barro, y techos de paja de dos aguas. También se han conservado muchos montículos artificiales o yácatas, basamentos piramidales y plataformas de piedra pulimentada que servían para asentar los templos. Igualmente construyeron empalizadas de troncos y bejucos, fosos abrasivos y algunas obras hidráulicas simples. La población de los sitios conocidos en la Cuenca de México se estima en unos 3000 a 4000 habitantes al principio de la revolución urbana -número que contrasta notablemente con los 200 habitantes calculados para las poblaciones neolíticas - y luego aumentó enormemente, cuando se constituyeron las grandes concentraciones metropolitanas.

La realización de la revolución urbana requirió una acumulación de capital, principalmente en la forma de artículos alimenticios. Y esta acumulación tuvo que ser concentrada después, para hacerla aprovechable con propósitos sociales. A la vez, el mejoramiento de las técnicas de cultivo y el aumento de la productividad del trabajo hicieron que el hombre se apegara cada vez más a la tierra, con la consiguiente aglutinación de las poblaciones urbanas. Por otra parte, el desenvolvimiento de la agricultura requirió una cooperación mayor y trajo como consecuencia la intensificación del trabajo colectivo; pero, al mismo tiempo, hizo que la posesión de la tierra adquiriera un carácter permanente $\mathrm{y}$, por lo tanto, formó el germen de la propiedad privada y de la conversión ulterior de la administración en un poder coercitivo. En la Cuenca de México, las nuevas técnicas agrícolas que se introdujeron, junto con el cultivo generalizado del maiz, consistieron en el aprovechamiento de los lagos mismos - y no sólo de sus riberas - con la construcción de las chinampas, y el uso de abonos para fertilizar el suelo. Ambas técnicas representan faenas colectivas que requieren la cooperación de grandes grupos, la planeación del trabajo y la dirección de una minoría o de un individuo. Desde un principio, la organización del clan matrilineal, con clara preponderancia de la mujer, empezó a ceder su lugar al clan totémico, en el cual quedaron equiparados el hombre y la mujer. Sin embargo, los datos 
arqueológicos disponibles no permiten saber cuáles eran entonces las formas de parentesco reconocidas y, por consiguiente, no sabemos en detalle cómo era la organización de dichos clanes. Con todo, es consecuente considerar - con apoyo en los vestigios de esta organización que subsistieron hasta la época histórica_- que el clan tenía sus dirigentes, elegidos voluntariamente por el prestigio personal adquirido en la administración o en las guerras; y que, después, su autoridad fue traspasada a los sacerdotes-hechiceros.

La economía urbana impulsó decididamente el desarrollo de la producción agrícola, haciendo aumentar todavía más los excedentes acumulados. Los cultivos preponderantes fueron el maíz, el frijol, la calabaza y el chile. Como consecuencia del incremento de la producción se acentuó la división del trabajo y se hizo posible la existencia de artesanos especializados en un solo oficio. Así se empezaron a distinguir claramente los canteros, albañiles, alfareros, lapidarios, joyeros y administradores; y, después, los comerciantes, jefes políticos, sacerdotes y sirvientes. Todos estos especialistas se mantenian gracias al excedente obtenido por el mayor rendimiento del trabajo de los agricultores, los cazadores y los pescadores. La nueva clase de los artesanos creada por la revolución urbana, al quedar liberada de la producción de alimentos, perdió también su apego al suelo y, lo que es más, debilitó sus vinculos tribales, sin adherirse con firmeza a los estados locales nacientes. Por ejemplo, la alfarería se manufacturaba casi siempre de manera local, pero utilizando técnicas, procesos, formas y diseños de carácter común. Se han podido advertir claramente diversas migraciones de grupos de alfareros especializados. Este fenómeno se explica por la incapacidad de una sola comunidad urbana para mantener un numeroso cuerpo de especialistas; $y$, por consiguiente, lo que se desarrolló fue un patrón de especialización de tiempo completo, sobre bases migratorias y de intercambio. Así, los artesanos iban a donde se les ofrecía ocupación conveniente; o bien, si eran esclavos, se les enviaba como mercancías a los lugares en donde su destreza se pagaba mejor. Este movimiento migratorio explica la rápida propagación de los procedimientos técnicos desarrollados por la revolución urbana.

Posiblemente la guerra fue la que hizo que se consumara la revolución urbana, con el consiguiente dominio de un pueblo sobre otros y la correspondiente concentración de los productos acumulados. Pero no siempre fue la conquista el único medio de esa consumación, sino que en otras ocasiones fue el resultado de la administración sacerdotal que acumuló y concentró el capital. En todo caso, la guerra ayudó a que se hiciera el descubrimiento de que el hombre podía ser domesticado al igual que los animales. Los prisioneros fueron sometidos a la esclavitud, en vez de sacrificarlos, debido a que la productividad de su trabajo rendía un excedente con respecto al consumo necesario para mantenerlos en condiciones de trabajar. La importancia de este descubrimiento es comparable a la de la domesticación de los 
animales $y$, sin duda, la esclavitud fue una de las bases de la economía urbana y un instrumento poderoso para la acumulación de capital. No obstante, no fue la guerra la única fuente para proveerse de esclavos. También los miembros más pobres y débiles de la comunidad se vieron obligados a someterse a la esclavitud - primero temporalmente y después por toda su vida y la de sus descendientes - a cambio de obtener el sustento o la protección de los miembros más prósperos. Igualmente fueron aceptados como esclavos los exiliados de otras comunidades.

Con la realización de la revolución urbana se produjo una acumulación mayor de capital, creció notablemente el intercambio comercial y se acentuó la singularización relativa de las culturas. Pero, al mismo tiempo, ya fuera por la guerra o por la aculturación pacífica, el hecho es que la revolución urbana tuvo una gran fuerza de propagación y, por ello, en lo que se refiere al régimen económico y sus consecuencias sociales, sirvió para homogeneizar la secuencia en el desenvolvimiento de las culturas del México Antiguo. En esta época fue cuando se inició la arquitectura de piedra, que pronto adquirió un carácter monumental, como lo testimonian la Pirámide del Sol en Teotihuacán, la pirámide decorada con insectos pintados de Cholula, las cabezas grandiosas de La Venta, el Observatorio de Monte Albán y el Templo E-VII-Sub de Uaxactún en la República de Guatemala. Por otra parte, como . ya lo hemos dicho, la cerámica se hizo mucho más compleja, tanto en sus técnicas como en sus colores y sus decorados. En la Cuenca de México, el primer foco destacado de la revolución urbana fue Tlatilco, que posiblemente estuvo asociado con la cultura olmeca, que se desarrolló en la faja costera del Golfo de México, desde la desembocadura del Papaloapan hasta Ciudad. del Carmen, comprendiendo el sur de Veracruz y Tabasco. Esta cultura olmeca, cuyas fases principales de La Venta y Tres Zapotes Medio florecieron aproximadamente entre los años 800 y $4^{00}$ a.n.e., representa indudablemente la primera manifestación del esplendor de la sociedad urbana en el México Antiguo. La consumación olmeca de la revolución urbana permitió que ésta se extendiera a otras regiones, cuando en ellas surgieron las condiciones económicas indispensables; fundamentalmente la adaptación del maíz para su cultivo en suelos y climas diferentes a los de las costas tropicales. Se han establecido efectivamente relaciones entre la cultura olmeca y varios núcleos de difusión de la revolución urbana en la Cuenca de México, los Estados de Morelos, Oaxaca, Guerrero, Puebla, la vasta región maya y, posiblemente, hasta las Repúblicas de Panamá y Costa Rica.

\section{Las consecuencias culturales}

La revolución urbana fue el resultado de la acumulación laboriosa de un conjunto importante de conocimientos científicos - topográficos, geoló- 
gicos, astronómicos, químicos, zoológicos y botánicos-, de experiencias obtenidas en la agricultura y las artesanías, y de la destreza práctica adquirida en esos trabajos. Todo esto fue aplicado con eficacia creciente a la producción, aumentando enormemente su rendimiento. Además, como consecuencia del comercio, las migraciones y las conquistas, se propagaron ampliamente las ciencias, las técnicas, las creencias y la nueva organización social. Una vez consumada, la revolución propició la invención de un nuevo método para transmitir las experiencias acumuladas y la ciencia aplicada, y de organizar y precisar mejor los conocimientos adquiridos. La ciencia y las técnicas requeridas para que la revolución se iniciara se habían transmitido en la forma de un saber artesanal, por medio del precepto oral y del ejemplo directo. Pero las necesidades prácticas impuestas por la nueva economía hicieron que la revolución urbana trajera aparejados los comienzos de la escritura, de la matemática, de la astronomía y del establecimiento de normas para medir, pesar y cambiar los artículos producidos para el comercio incipiente.

En la medida en que aumentó la riqueza producida por la consumación de la revolución urbana, fueron creciendo también las complicaciones de su administración, haciendo que esta tarea se convirtiera en un trabajo especializado y de tiempo completo. A la vez resultó imposible seguir confiando en la memoria o en los signos empleados individualmente como recordatorios, para llevar las crónicas y cuentas de la administración. Entonces se hizo necesario establecer un sistema de signos convencionales aceptados y autorizados por la sociedad, que constituyó el principio de la escritura. De, este modo, los registros se hicieron inteligibles para todos los conocedores de la convención establecida. Con la escritura de las palabras se produjo una verdadera revolución en la transmisión del conocimiento, ya que valiéndose de ellas fue como el hombre pudo inmortalizar su experiencia y comunicarla directamente a sus contemporáneos lejanos y a las generaciones subsecuentes. Sin duda, los signos escritos constituyeron el primer paso para que la ciencia pudiera superar los límites de lugar y de tiempo. Sin embargo, al principio, la escritura fue un arte sumamente difícil y especializado, que requería un largo aprendizaje. En realidad, sólo unos cuantos gozaban del ocio necesario para penetrar los secretos del arte de leer y escribir; y, al mismo tiempo, quienes conseguian dominarlo se convertían en funcionarios de un servicio público organizado y permanente. De hecho, se trataba de un oficio como el del alfarero, el tejedor o el guerrero; pero pronto se convirtió en una profesión privilegiada y sus practicantes, en vez de considerarla como una clave para la adquisición del conocimiento científico, la tomaron como un instrumento para prosperar y hacerse de una posición social elevada, bajo la protección de los sacerdotes a cuyo servicio se encontraban.

La matemática fue una consecuencia de las necesidades económicas crea das por la revolución urbana, de una manera tan obvia como lo fue la esr 
tura. La administración de los productos y las transacciones comerciales requirieron del establecimiento de patrones fijos para pesar y medir, de un sistema de notación numérica y de reglas para la ejecución de las cuentas. El primer paso para el desarrollo del arte de calcular fue la invención de un sistema de símbolos, mediante el cual se pudieron escribir en forma abreviada todos los números, para los cuales ya existían nombres en el lenguaje hablado. El paso siguiente fue el de simplificar las operaciones que ya se realizaban en forma rudimentaria. La suma y la resta son simplemente formas abreviadas de la técnica de contar los objetos uno por uno. Y, por su parte, la multiplicación y la división son sencillamente procedimientos abreviados para sumar y restar cantidades iguales. Por otro lado, las necesidades prácticas de la agricultura impusieron la observación cuidadosa de los cuerpos celestes. En los cielos claros que predominan en las latitudes tropicales, los habitantes del México Antiguo pronto reconocieron la regularidad de los acontecimientos celestes y su conexión cronológica con los sucesos terrestres. Y lo que es más, los éxitos obtenidos en la predicción del tiempo oportuno para la realización de las faenas agrícolas, los animaron a proseguir dichas observaciones, con la vana esperanza de poder predecir así otros acontecimientos importantes en la vida de los hombres. De este modo, al legítimo propósito de fijar las fechas agrícolas y los festivales conectados con ellas, se agregó la elaboración de los pronósticos astrológicos que, aun cuando carecieron de valor intrínseco, fueron convertidos en instrumentos para el fortalecimiento de la autoridad política. Por otra parte, la aplicación del arte de contar a las observaciones astronómicas cada vez más precisas, produjo la formulación del calendario; primero con base en las lunaciones y después en correspondencia con el año solar. En todo caso, el calendario representa la primera conquista científica obtenida con fundamento en la observación y en la aplicación acertada del cálculo. A la vez, el calendario fue también la primera justificación del propósito científico de hacer predicciones precisas. Y esta primera aplicación de la predicción científica fue un factor importante para el reconocimiento de la autoridad de quienes tenían el dominio de los conocimientos necesarios para hacerlo, o sea, de los sacerdotes.

En un principio no habia una verdadera distinción en la forma de transmitir las ciencias aplicadas y las eruditas. La instrucción que se daba para aprender el arte de contar o el de curar, era prácticamente similar a la que se daba al aprendiz de alfarero o de tejedor. El discípulo observaba el trabajo de su maestro, quien le mostraba la manera de ejecutar las operaciones y, luego, el aprendiz se ponía a trabajar bajo la dirección del maestro, quien le corregía los defectos de ejecución. Pero, con el desarrollo de la sociedad urbana, las nuevas artes de escribir, contar y observar los astros se convirtieron en profesiones "respetables", y sus practicantes se asociaron directamente a la clase dirigente; en contraste con los artesanos y agricultores, cuyo trabajo 
manual empezó a ser considerado como despreciable. Pronto se estableció una diferenciación notable entre el saber artesano - que no se transmitía por escrito- y la tradición literaria que se fue estableciendo en algunas ciencias y seudociencias. Las ciencias aplicadas - como la botánica, la química, la mineralogía y la geología - quedaron incluidas en la tradición oral de los artesanos; mientras que las matemáticas, la medicina, la cirugía, la astrología, la alquimia y la adivinación, sirvieron de tema a tratados escritos. De esta manera se formó un cuerpo de disciplinas eruditas, sólo accesibles para quienes estaban iniciados en los misterios de la escritura y la numeración.

Entre los conocimientos concretos que tuvieron los antiguos mexicanos en la época de la revolución urbana, podemos agregar la distinción cada vez más acertada entre las plantas comestibles y las venenosas, lo mismo que de otras plantas utilizadas para diversos menesteres, la fijación precisa de las épocas más propicias para la recolección de los productos silvestres; la observación de las costumbres de los animales que les interesaban; la determinación de las rutas y caminos más convenientes; el desarrollo de algunas formas de navegación lacustre, fluvial y marítima; el mejoramiento de las técnicas de cultivo; la observación esmerada de las condiciones meteorológicas; el estudio de las propiedades de los materiales que empleaban; y el perfeccionamiento de sus instrumentos de trabajo. Además, en esta época surgen los sistemas de escritura jeroglífica; se establece el avanzado sistema de numeración vigesimal, que los mayas desarrollaron prodigiosamente, incluyendo la concepción del cero y la atribución de un valor de posición a las cifras; se forma el calendario ceremonial de doscientos sesenta dias; y aparece el culto organizado, con templos y jerarquías sacerdotales. $Y$ todo esto estuvo cimentado en el establecimiento de una organización social apropiada para la concentración y la administración de los excedentes alimenticios producidos; la estratificación social con base en la posición ocupada en las relaciones económicas; el dominio de una clase — la sacerdotal - sobre los medios de producción; la guerra organizada como instrumento de dominio económico y político; y el desenvolvimiento de los centros urbanos sostenidos con la renta de la tierra, los tributos y el comercio.

Ahora bien, la revolución urbana no fue trasplantada simplemente de un centro a otro, sino que cada uno de ellos constituyó un desarrollo orgánico basado en sus propias transformaciones económicas y en las innovaciones tecnológicas. De una manera analógica, se puede comparar con el surgimiento de la industria mecanizada en el siglo xviII, que se produjo en aquellos países europeos que compartían una tradición científica, cultural y económica común. Con frecuencia, la revolución se propagó por medio de la violencia de la guerra, imponiéndose a través del dominio de los conquistadores. Sin embargo, algunas comunidades estaban demasiado atrasadas para aprovechar las ventajas de la nueva eronomía y sus productos. Por otra parte, 
también hubo comunidades que pudieron resistir venturosamente los ataques o las amenazas, pero únicamente sobre la base ineludible de asimilar parcialmente la civilización de los agresores. En todo caso, la aculturación provocada por las invasiones, las emigraciones y los contactos comerciales, fue el cauce seguido para la propagación de la revolución urbana y el establecimiento de la civilización. Sólo que, en la medida en que tuvo éxito el ajuste entre la nueva organización social y las condiciones económicas que le servían de apoyo, las comunidades urbanas tendieron a hacerse conservadoras. Además, si bien la revolución urbana se realizó como resultado de las grandes contribuciones hechas al conocimiento científico y sus aplicaciones, en cambio, las transformaciones producidas en la organización social, junto con el menosprecio hacia las artes manuales y la exaltación desorbitada y misteriosa de los oficios literarios, trajeron consigo la deformación del avance científico y el retardo en el progreso técnico. Así, contrastando con el desarrollo logrado antes de la revolución urbana e inmediatamente después de ella, la civilización no vino a ser la aurora de una nueva época de avance acelerado, sino más bien la culminación y luego la detención del anterior periodo de crecimiento. Y una explicación parcial de esta retardación en el ritmo del progreso social la tenemos en las contradicciones internas que la propia revolución urbana suscitó en el seno de las sociedades civilizadas.

\section{La civilización clásica}

La consumación de la revolución urbana se manifiesta en el establecimiento de centros de civilización, con un sistema ceremonial complicado, sustentados en comunidades subsidiarias; y en los cuales se muestra una organización compleja, una división del trabajo notable y una indudable estratificación social jerarquizada. La primera sociedad civilizada que surge en el México Antiguo es la de La Venta, en el Estado de Tabasco, que tuvo su apogeo entre los años 800 y $4^{\text {oo }}$ a.n.e., cuando apenas se estaba realizando en otras partes la revolución urbana y la mayoria de las comunidades tenían todavía una economía neolítica. Entre los restos conservados de esta cultura de La Venta tenemos las primeras inscripciones calendáricas. Además, desde entonces se muestran las características elementales que luego se hicieron peculiares de los centros urbanos, cuando éstos se generalizaron en el México Antiguo. Dichos centros, constituidos por grandes construcciones destinadas a la celebración de ceremonias, fungieron como núcleos regionales integradores y coordinadores. Estaban gobernados por una casta sacerdotal auxiliada por numeroso personal sustraído a la actividad productiva, que se sustentaba en un sistema de producción mucho más eficaz. Los grandes templos, los monumentos y las plazas enormes, desproporcionados para la magnitud aparente de la población constante que puede colegirse de las 
habitaciones civiles relativamente escasas, correspondian bien a sus funciones como centros comerciales, religiosos, administrativos, políticos y sociales de un gran número de comunidades rurales dependientes, situadas a su alrededor. El centro ceremonial, con su solemne planificación urbana, servía de asiento al sacerdocio $y$, por lo tanto, representaba el gobierno teocrático que dirigía y coordinaba a la sociedad en todos sentidos. Era el lugar de concentración periódica para celebrar las ceremonias rituales y otros actos de interés colectivo, como el tianguis o mercado que se efectuaba una vez cada cinco días -y posteriormente una vez a la semana- para que la población campesina intercambiara sus productos. En rigor, este patrón del centro ceremonial se mantuvo hasta la época de la conquista; y, lo que es más, algunas de sus instituciones persisten hasta nuestros dias.

Los testimonios existentes no permiten atribuir la propagación de la civilización clásica a una fuente en particular, sino al desarrollo económico, social, político y científico de la mayoría de las comunidades. Sin embargo, en el México Antiguo -como ocurrió en realidad en las otras regiones del mundo- no hubo una absoluta homogeneidad en el desarrollo, ni menos una correspondencia cronológica inflexible en sus distintas partes. Por el contrario, siempre existieron núcleos de cultura más avanzada y regiones marginales menos desarrolladas. Al principio de la civilización, los núcleos estuvieron en el sur, siendo entonces marginal la Cuenca de México; pero sin que hubiera una coincidencia completa desde el punto de vista cronológico entre los desenvolvimientos de La Venta, y los de Monte Albán y la región maya. Después, al difundirse la civilización, Teotihuacán se convirtió en el centro principal en la Mesa Central, y la cultura maya se desplazó hacia el Petén. Con el florecimiento de la civilización clásica se acentuaron las coincidencias culturales que dieron unidad al México Antiguo, a pesar de las diferencias que distinguen sus diversas manifestaciones. Desde luego, podemos señalar en esta época, por su desarrollo específico: la cultura maya en Guatemala, Honduras, Chiapas y Yucatán; la cultura zapoteca en Oaxaca; la cultura mixteca, también en Oaxaca; la cultura olmeca en Tabasco y Veracruz; la cultura totonaca en Veracruz; la cultura tarasca en Nayarit, Colima, Jalisco, Michoacán y Guerrero; además de otras culturas menos desarrolladas del norte y el oeste. Por lo tanto, considerando que la época de la civilización clásica abarca aproximadamente del año 400 al 900 de nuestra era, quedan comprendidos en ella: el Primer Periodo Maya (317-633 n.e.), que corresponde a las tres fases de la cerámica de Tzakol; el Periodo Medio Maya (633-731), correspondiente a la fase antigua de la cerámica de Tepeu; la primera parte del Gran Periodo Maya (73 $1-889)$, en la parte correspondiente a la fase media de la cerámica de Tepeu; Teotihuacán II, III y IV; Monte Albán IIIA y IIIB; Yucuñudahui, en Oaxaca; Pavón III y IV, en la Huasteca; El Tajín, de la cultura totonaca en Veracruz; Chametla I y II, 
el Complejo de Huatabampo, Tuxcacuexco y Los Ortices, en el occidente; Delicias, Jiquilpan y Apatzingán, en Michoacán; la Fase de Ixtlán; Xochicalco en Morelos; y Tamazulapan, de la cultura mixteca en Oaxaca.

Dentro de la organización de los centros urbanos clásicos, el manejo de las funciones políticas, administrativas y religiosas quedó concentrado en el sacerdocio, que se organizó poderosamente como clase dirigente. Los templos fueron también lugares para el almacenamiento de los productos, y el control de los bienes y recursos llevó al establecimiento y el desarrollo del comercio. Desde el punto de vista económico, se acentuó notablemente la especialización del trabajo; y los centros urbanos tuvieron artesanos y otros especialistas de tiempo completo, ligados directamente a las exigencias del ceremonial religioso, la administración de la producción y las necesidades del gobierno. Y el mantenimiento de estos trabajadores, lo mismo que de los sacerdotes gobernantes y sus servidores, requería de grandes cantidades de provisiones agrícolas y de materias primas para los oficios, lo cual estimuló el comercio y creó la necesidad de establecer rutas comerciales. Los contactos entre los diversos centros urbanos deben de haberse hecho principalmente entre las clases dominantes -en la forma de intercambios mercantiles, tecnológicos y científicos- y a través de los artesanos y servidores. Por su parte, el sacerdocio dirigió las grandes obras de urbanización y de construcción, manejó los conocimientos matemáticos y astronómicos fundamentales para la agricultura, administró la realización de los trabajos y la acumulación de los productos - satisfaciendo así los intereses sociales de especialización y concentración- y adquirió con todo esto el dominio de la sociedad.

Durante el periodo de la civilización clásica se desenvolvió pacíficamente el intercambio comercial y cultural entre los centros urbanos que compartieron así la cultura común del México Antiguo. En el dominio social, el surgimiento de la teocracia inició el proceso de desaparición de la organización en clanes; aunque, como hemos dicho, muchos elementos de esa organización persistieron hasta el final del México Antiguo. Desde el punto de vista tecnológico, se introdujo entonces el uso de moldes para las piezas de cerámica, y se desenvolvieron la lapidaria, la plumaria y el trabajo del jade y la turquesa. En los centros urbanos se construyeron muchos palacios, pirámides, templos y tumbas; en particular, la arquitectura funeraria se distinguió por las tumbas cruciformes. Apareció el culto a Quetzalcóatl - la divinidad civilizadora - en constante asociación con Tláloc —el dios de las lluvias-. En Teotihuacán se puede advertir claramente que, además de los barrios correspondientes a los clanes totémicos, existian también barrios de artesanos; lo cual revela la profundidad que había alcanzado la división social del trabajo. También Teotihuacán es la primera gran productora de máscaras de piedra del México Antiguo, aunque no fue la iniciadora. Sin embargo, en Teotihuacán no existen estelas, ni se encuentra la bóveda maya, ni el cero, ni el 
juego de pelota. Además, es sorprendente el número tan reducido de ins: cripciones jeroglíficas que existen allí; sobre todo en comparación con el gran número de inscripciones mayas de la misma época y con las relativamente numerosas del Valle de Oaxaca. Por otra parte, Teotihuacán fue también un gran centro productor de una multitud de objetos de cerámica y de diversas piedras; y en ella se hicieron grandes progresos en la arquitectura, la escultura y la pintura. Teotihuacán fue siempre una ciudad abierta, sin ninguna construcción defensiva, debido seguramente a que no había peligro de que fuera atacada. Mediante el intercambio comercial, los teotihuacanos esparcieron su influencia de un extremo al otro del México Antiguo, enviando piezas de cerámica y objetos diversos, algunos de los cuales se han encontrado en las tumbas de Monte Albán en Oaxaca, en Kaminaljuyú en el altiplano de Guatemala, en Veracruz, en el occidente y aun entre los pueblos menos desarrollados del norte.

Dentro del desarrollo de la cultura maya, durante el Primer Periodo tenemos la iniciación de los monumentos de piedra; el surgimiento y evolución de la policromía; la erección de estelas de piedra, entre las cuales se distinguen especialmente la 9 de Uaxactún (328 n.e.), la 5 de Balakbal (4o6 n.e.), la I de Uolantún (409 n. e.), la I de Tulum $\left(5_{6} 6_{4}\right.$ n. e.) y la I de Ichpaatún ( 593 n. e.). También se elaboró entonces la famosa placa de Ley. den, labrada en jade en Tikal (320 n. e.); se construyó el dintel de Oxkintok (475 n.e.); se desarrolló la bóveda de piedras voladizas; y se introdujo la cultura maya en el norte de Yucatán, difundiéndose enormemente. Durante el Periodo Medio se establecieron nuevos centros ceremoniales, como Palenque (64o n.e.), Yaxchilán (692 n.e.) y Chakanputún (731 n.e.), a la vez que se abandonaron otros, como Chichén Itzá (en 692 n.e.), y se consolidó la cultura. En la primera fase del Gran Periodo hubo un gran florecimiento de la cultura maya, sobre todo en el sentido de su sistematización; mientras que, en la segunda fase, se inició la decadencia y luego se produjo el colapso.

\section{La revolución secularista}

Tal como lo hemos expresado, la revolución urbana tuvo como base la acumulación de la riqueza resultante del mejoramiento de las técnicas agricolas, de la generalización del cultivo de algunas plantas - principalmente del maíz-, de la extensión del cultivo de otras —como el cacao y el algodón - que se hicieron productos para el cambio, y la consiguiente concentración de la riqueza en manos de la clase sacerdotal gobernante y de otros grupos sociales auxiliares y dependientes de ella. Esta concentración fue fundamental para asegurar la producción de los recursos excedentes requeridos y para hacerlos aprovechables y utilizables socialmente, de un modo efec- 
tivo. Pero, en la práctica, la concentración de la riqueza implicó también la degradación económica y social de la inmensa mayoría de la población. La situación de los productores directos - agricultores y artesanos - mejoró realmente con las obras públicas llevadas a cabo y con la regular seguridad garantizada por el gobierno teocrático. Sin embargo, su participación material en la nueva riqueza fue mínima y, desde el punto de vista social, los agricultores se hundieron hasta quedar colocados en la condición de arrendatarios, o incluso de siervos y esclavos. Por su parte, los artesanos tampoco tuvieron mucha participación en la distribución de la riqueza, ya que su situación social fue semejante a la de los otros trabajadores manuales, y muchos de ellos quedaron reducidos a la esclavitud. Además, los sacerdotes tenían pocos incentivos para promover la invención, ya que disponían de reservas casi ilimitadas de trabajadores $y$, por lo tanto, no tenían necesidad de molestarse en fomentar el progreso técnico para ahorrarse la mano de obra. Por otro lado, la separación establecida y mantenida firmemente entre los trabajadores manuales y los intelectuales, hizo que el progreso técnico fuese sumamente lento en la práctica, en el sentido de la invención y la incorporación de nuevos procedimientos e instrumentos. Como consecuencia, las sociedades teocráticas surgidas de la revolución urbana se vieron envueltas en una serie de contradicciones internas irremediables. Por ello, después de alcanzar su esplendor en un tiempo relativamente corto, tuvieron una decadencia súbita.

El colapso de las sociedades teocráticas adoptó la forma de una crisis, debido a que obedeció a factores internos de la propia estructura social. Su desarrollo económico y cultural descansó en la opresión de la clase sacerdotal sobre la población trabajadora. Por ello, el sistema condujo a la miseria de los productores y la agudización de los contrastes sociales. Por último, la opresión acabó por ser insoportable y provocó el debilitamiento de los vínculos internos, y el régimen teocrático se derrumbó. En algunas partes, como en Teotihuacán, se han conservado testimonios de que la destrucción fue muy violenta; mientras que en la mayoría de los centros mayas no existen indicios de saqueos o de incendios. Pero, de una manera o de otra, lo cierto es que desaparecieron los centros ceremoniales de la civilización clásica, que servían de núcleo a las sociedades teocráticas. Para explicar su desaparición se han aducido varias causas: guerras, epidemias, sequías, terremotos, erupciones volcánicas, cambios climáticos, agotamiento de la tierra y hasta motivos religiosos o supersticiosos. Es posible que uno o varios de estos factores hayan intervenido en algunos casos, aunque siempre de manera secundaria o como consecuencia de la causa principal. Porque el hecho de que los agricultores hayan seguido viviendo alrededor de los centros ceremoniales extinguidos, demuestra que su desaparición se debió a una transformación política y social interna. Lo que ocurrió fue que la clase sacerdotal fue despojada 
del poder por una revolución secularista, encabezada por caudillos militares. A partir de entonces, los sacerdotes quedaron supeditados a los guerreros y, al mismo tiempo, tuvieron que cederles en buena parte su prestigio religioso: Por otro lado, la revolución antiteocrática nos permite entender la pérdida de algunos conocimientos científicos que eran mantenidos en secreto por los sacerdotes y que no fue posible arrancarles, como sucedió con la llamada cuenta larga maya. La revolución secularista también nos aclara los incendios de Teotihuacán y las mutilaciones que se advierten en los frescos de Bonampak, en las figuras que representan a los sacerdotes dirigentes; y explica igualmente el hecho de que los campesinos siguieran viviendo y cultivando la tierra en torno de los templos abandonados. Más aún, los mitos toltecas acerca de las luchas entre Quetzalcóatl y Tezcatlipoca, que terminan con la derrota y la huida de Quetzalcóatl, seguramente reflejan legendariamente la pugna entre los sacerdotes y los militares por el dominio del poder político, económico y social.

Teotihuacán se abatió, pero sus tradiciones culturales fueron asimiladas por las civilizaciones posteriores, aunque con modificaciones y reinterpretaciones. Una parte de sus habitantes emigró a otros sitios de la Cuenca de México, principalmente a Azcapotzalco, en donde los testimonios arqueológicos indican una fase epigonal de la cultura teotihuacana. En todo caso, las escasas exploraciones que se han hecho en Azcapotzalco no han puesto al descubierto ninguna construcción de importancia en esa época. Posteriormente, la historia teotihuacana se convirtió en un mito. En la lengua de toltecas y aztecas, Teotihuacán significa "el lugar en que vivían los antiguos", o "el sitio de los dioses". Llevados por la impresión que les producian los majestuosos edificios teotihuacanos, los toltecas atribuyeron su construcción a una especie de gigantes, los quinametzin; y, como prueba "objetiva" de la verdad de su existencia, tuvieron a los restos fósiles de algunos grandes animales desaparecidos. Los huesos del mamut sirvieron así de confirmación del quinametzin mítico; y todavía los primeros cronistas españoles enviaron a Carlos V un fémur de mamut para convencerlo de la talla alcanzada por los ancestros de los conquistados. El desplazamiento de la cultura teotihuacana a Azcapotzalco debe de haber servido de antecedente para el florecimiento que más tarde tuvo allí la cultura tepaneca. Además, no todas las ciudades desaparecieron, sino que algunos sitios de la civilización clásica se mantuvieron dentro de la nueva organización secular y sirvieron de enlace directo con la nueva sociedad militarista de la época histórica. El ejemplo más interesante de la Mesa Central es el de Xochicalco, en el Estado de Morelos, en donde se conservó el culto teotihuacano a la serpiente emplumada, que después fue adoptado en buena parte del México Antiguo. El hecho de que Xochicalco se mantuvo en esa época lo tenemos comprobado por el juego de pelota con anillos, que es igual al de Tula y seguramente su contemporáneo, lo mismo 
que en el gran número de otros objetos que también corresponden a los de Tula, por su estilo y la fecha de su elaboración. Además, en una eminencia del terreno cercana a la pirámide de Xochicalco se encuentra una fortaleza, que constituye el testimonio más antiguo que se tiene en la meseta central de la preocupación por los posibles ataques, que se hicieron muy frecuentes con la constitución de los estados militaristas.

En el Estado de Veracruz hubo otro centro importante, que fue el Tajín, cuya pirámide principal —formada por siete cuerpos_ es una de las construcciones más impresionantes del México Antiguo. Sus constructores -que fueron posiblemente los pipiles - alcanzaron una expansión considerable, sobre todo al final de la época de la civilización clásica. Se han encontrado algunos objetos procedentes del Tajín entre las ruinas del palacio de Palenque, lo cual indica que posiblemente hayan conquistado ese sitio los pipiles. En cambio, su influencia sobre Tula no fue muy notable, porque entonces el Tajín ya se encontraba en decadencia. Por lo demás, su desaparición fue tan completa que, en la época de la conquista, nadie advirtió su existencia; y sólo hasta fines del siglo xviI fue cuando José Antonio Alzate mencionó el Tajín por primera vez. Por su parte, los centros mayas del Petén siguieron erigiendo estelas de piedra y construyendo edificios hasta los últimos años del siglo Ix. Por otro lado, los zapotecas de Monte Albán se convirtieron en los grandes arquitectos del México Antiguo, cubriendo los valles del Estado de Oaxaca con multitud de edificios. También en esa época se siguió elevando la pirámide de Cholula en el valle de Puebla. Pero, entre los últimos años del siglo Ix y los primeros del siglo $\mathrm{x}$, la revolución antiteocrática se propagó a todos los centros ceremoniales del México Antiguo, acabando con la organización social que les servía de base. Entonces se extinguieron igualmente los grandes centros urbanos de la región central maya, en la última parte del Gran Periodo (889-987), y se produjo también el colapso de Monte Albán.

Con la desaparición del dominio sacerdotal y el surgimiento de los guerreros como clase gobernante, se consumó la revolución secularista que trajo consigo una nueva organización política de la sociedad. Desde luego, se produjo una disminución en la hipertrofia de la función ceremonial y las sociedades militaristas se orientaron hacia un mayor equilibrio urbano. Los guerreros constituidos en gobierno desempeñaron funciones económicas fundamentales para el desarrollo social. Se preocuparon principalmente porque los antagonismos engendrados por la revolución urbana - sobre todo, las luchas entre las clases con intereses en conflicto- no llevaran a la situación de que las clases y la propia sociedad se consumieran en un combate estéril. Los grandes gobernantes militares se jactaron de sus actividades económicas, como fueron la construcción de canales y diques, y la edificación de templos y palacios. Es indudable que el poder militarista aceleró la acumulación de capital, en forma de alimentos y otras riquezas; y con los excedentes así obte- 
nidos se mantuvieron los funcionarios, comerciantes y hombres de armas. Mientras tanto, los militares cumplieron bien la función económica de proteger las ciudades, sus canales, sus campos cultivados y sus comunicaciones, contra las incursiones de otros pueblos menos prósperos. En fin, el dominio de los guerreros acabó por crear un orden político más compatible con las realidades económicas de la sociedad urbana. Y, por lo demás, las nuevas sociedades militaristas surgidas de la revolución antiteocrática se preocuparon por llevar cuidadosamente las crónicas de los sucesos que consideraron más importantes, terminándose así la prehistoria del México Antiguo al comenzar la época de la historia escrita.

ELI DE GoRTARI

\section{BIBLIOGRAFIA}

Pedro Armillas, Cronologia y periodificación de la historia de América Precolombina, Sociedad de Alumnos de la Escuela Nacional de Antropología e Historia, Suplemento de la Revista Tlatoani, México, 1957.

_- Programa de Historia de la América Indigena, Primera Parte: América Precolombina, Unión Panamericana, Estudios Monográficos II, Washington, 1957.

Ignacio Bernal, Mesoamérica, Periodo Indigena, Instituto Panamericano de Geografía e Historia, Programa de Historia de América, I.4, México, 1953.

- Tenochtitlán en una isla, Instituto Nacional de Antropologia e Historia, México, 1959.

-y G. Reichel-Dolmatoff, Mesoamérica y Colombia (Suplemento), Instituto Panamericano de Geografía e Historia, Programa de Historia de América, I.4 y 6, México, 1953.

Alfonso Caso, "New World Culture History: Middle America", Anthropology Today, An Encyclopedic Inventory, The University of Chicago Press, Chicago, 1953, págs. 226-237.

V. Gordon Childe, Los origenes de la cizilización, Fondo de Cultura Económica, México, 1954 .

Kunz Dittmer, Etnologia general, formas y evolución de la cultura, Fondo de Cultura Económica, México, 1960.

Federico Engels, El origen de la familia, la propiedad privada y el Estado, Ediciones en Lenguas Extranjeras, Moscú, 1952.

Clarence L. Hay, et al., The Maya and their neighbors, O. Appleton-Century, Nueva YorkLondres, 1940.

Paul Kirchhoff, "Mesoamérica: sus límites geográficos, composición étnica y caracteres culturales", Acta Americana, Vol. I, Núm. I, México, 1943.

Sylvanus G. Morley, La civilización maya, Fondo de Cultura Económica, México, 1947.

Julio César Olivé Negrete, "Estructura y dinámica de Mesoamérica", Acta Anthropologica, Época 2, Vol. I, Núm. 3, México, $195^{8}$.

Angel Palerm, "La secuencia de la evolución cultural en Mesoamérica", Boletin Bibliográfico de Antropologia Americana, Vol. XVII, Parte Primera, 1954, págs. 205-233.

- "La base agricola de la civilización urbana en Mesoamérica", Las civilizaciones antiguas del Viejo Mundo y de América, Unión Panamericana, Estudios Monográficos I, Washington, 1955 , págs. 29-44.

Raúl Pavón Abreu, "Cronologia maya", Los mayas antiguos, Monografías de Arqueología, Etnografía y Lingüistica Mayas, El Colegio de México, México, 1943. 
Román Piña Chan, Las culturas preclásicas de la Cuenca de México, Fondo de Cultura Económica, México, 1955.

Julian H. Steward, "Introducción: El Symposium sobre las civilizaciones de regadío", "Algunas implicaciones del Symposium", Las civilizaciones antiguas del Viejo Mundo y de América, Unión Panamericana, Estudios Monográficos I, Washington, 1955, págs. $1-5$ y $60-82$.

Mauricio Swadesh, Mapas de clasificación lingüistica de México y las Américas, Cuadernos del Instituto de Historia, Serie Antropológica, Núm. 8, U.N.A.M., México, 1959.

J. Eric S. Thompson, Grandeza y decadencia de los mayas, Fondo de Cultura Económica. México, 1959 .

George C. Vaillant, La civilización azteca, Fondo de Cultura Económica, México, 1944. 